\title{
Intraoperative imaging based on common-path time-domain reflectometry for brain tumor surgery
}

\author{
JAE-HO HAN $^{1 *}$, JAEPYEONG CHA ${ }^{2}$ \\ ${ }^{1}$ Department of Brain and Cognitive Engineering, Korea University, \\ 145 Anam Rd., Seoul 02841, South Korea \\ ${ }^{2}$ Sheikh Zayed Institute for Pediatric Surgical Innovation, Children's National Health System, \\ Department of Pediatrics, George Washington University School of Medicine and Health Sciences, \\ Washington, DC 20010 USA
}

*Corresponding author: hanjaeho@korea.ac.kr

\begin{abstract}
Minimally invasive intraoperative imaging plays a crucial role in delicate microsurgeries for precise operation monitoring in which fiber optic imaging can be considered as an endoscopy and surgical proximity guidance tool due to its compactness. This paper presents a near-infrared time -domain reflectometric common-path optical coherence tomography imaging technique using a bare-fiber probe mounted directly on a scanning galvanometer. The common-path setup allows the use of a freely adjustable optical path length and a disposable fiber probe, as well as eliminating the need for an additional dedicated reference optical path. Experimental results demonstrate clear discrimination between the brain tumor tissue and the normal tissue for mouse brains with the images acquired in real-time over a wide area. The proposed method enables real-time and in situ visualization of tumor resection for intraoperative imaging, and this study demonstrates the feasibility of its application to microsurgical interventions.
\end{abstract}

Keywords: optical coherence tomography, intraoperative imaging, reflectometry, endoscopy.

\section{Introduction}

Intraoperative imaging can help locate and visualize regions of interest during delicate surgical procedures, to supplement preoperative image data [1]. In particular, brain images acquired before surgery can prove to be a poor representation of the state of the brain during surgery because the position of the tumor continuously evolves in the period between image collection and surgical intervention [2]. By combining surgical tools with imaging probes, intraoperative imaging offers real-time visualization during surgery, and it enables surgeons to operate more precisely and with minimal reliance on microscope navigation in operating rooms [3] . As a candidate to overcome these 
challenges, a compact all-fiber optical coherence tomography (OCT), a minimally invasive biomedical modality that provides micrometer-scale subsurface cross-sectional imaging, can be utilized by inserting a fiber-based endoscopic imaging probe in small and delicate areas [4]. Even though appropriate disposable and compact fiber optic probes have been developed for various surgical purposes in advanced microsurgery and image-guided intervention, the design and fabrication of the probes require a complexed electro-mechanical scanning system using beam deflecting mirrors merged with optical fiber probes [ $[$ ].

In this work, time-domain reflectometry-based common-path OCT (CP-OCT) was employed, with an optical fiber attached to a scanner, as an intraoperative imaging tool to evaluate the extent of tumor resection. This method enables lesional resolution via real-time imaging, with image quality sufficient for viewing safely removed brain tumors in a small-animal model. In the following sections, the details of the experimental setup (Section 2) and the obtained results (Section 3) are shown with a followed brief summary in Section 4.

\section{Experiment}

The experimental setup is schematically shown in Fig. 1. The OCT system is constructed based on an all-fiber time-domain common-path interferometer (Optiphase HSR -3000-SW reflectometer) that performs depth scanning (A-scans) with a superluminescent diode (SLED) broadband optical source (40-nm FWHM bandwidth centered at $1320 \mathrm{~nm}$ ) []ㅡ. To achieve a common-path configuration, the distal end of the optical fiber probe should be vertically cleaved, very cleanly. Using the common-path configuration means that a separate arm for the reference signal is not required; hence, it is possible to use an arbitrary length of fiber. As a result, a compact and adjustable configuration, a desirable feature for intraoperative intervention, is achieved. Based

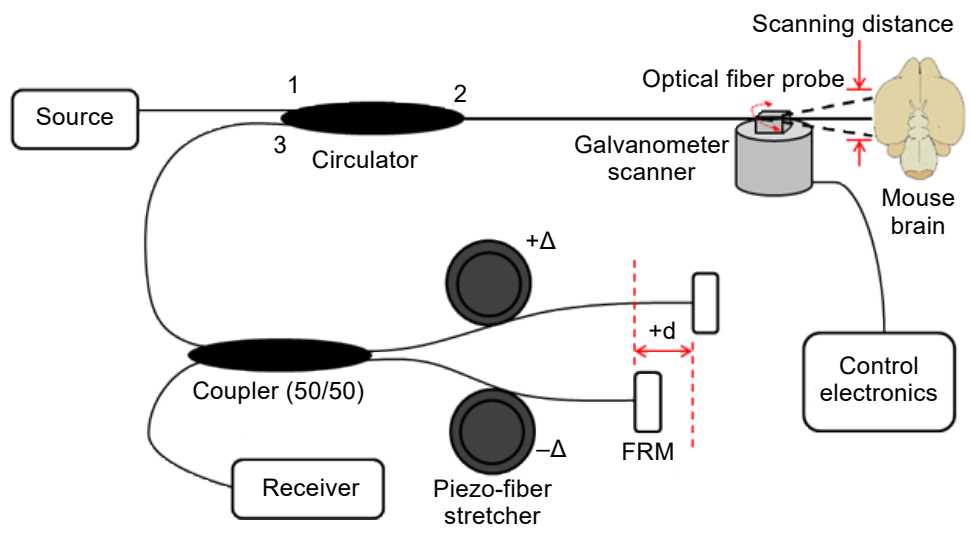

Fig. 1. Schematic of broadband near-infrared time-domain reflectometry with common-path configuration for intraoperative brain imaging. 
on the CP-OCT, the optical path for the reference and the signal are shared which enables a simpler optical configuration. Here, the reference is obtained by Fresnel reflection between the glass-air interface at the cleaved distal end of the optical fiber. Furthermore, this CP-OCT optical fiber probe functions both as transmitter and receiver for illuminating and collecting light to and from the specimen, respectively. Fiber optic autocorrelation was achieved by constructing a Michelson interferometer using a pair of push-pull piezoelectric fiber stretchers. Both the signal and reference beams were divided into two parts by a 50/50 coupler, and each part of the light was sent to a Faraday mirror (FRM). The reflected beams from the FRMs were combined and sent to an InGaAs p-i-n optical receiver to process the interference signals and reconstruct images.

In the present study, a bare-fiber probe, fabricated from a standard single-mode optical fiber (Corning SMF-28) with a cleaved end, was mounted and directly attached to a galvanometer (Nutfield Tech QS-7). The fiber probe swings back and forth, following the rotation of the galvanometer to achieve lateral scanning (B-scans). The galvanometer is driven by a control electronics servo chip (Nutfield Tech QD-1500) with a triangular wave input $(0-3 \mathrm{~V})$. A galvanometer mounted with reflection mirror is typically applied to control and deflect the beam for fast B-scanning. In the proposed system, the galvanometer was utilized to scan the fiber probe directly, and its imaging capability was demonstrated by fast scanning of a rodent (mouse) brain model with an implanted glioma in the frontal lobe. The scanning radius of the fiber was $57.5 \mathrm{~mm}$ from the tip to the axis of the galvanometer and the scanning length or arc angle for our system was approximately $0.039 \mathrm{rad} / \mathrm{V}$, as determined by the triangular-wave voltage. The scanning speed depends on the frequency of the triangular wave input, which in this system was in excess of $100 \mathrm{~Hz}$ during the acquisition of real-time $2 \mathrm{D}$ images. The distance between the probe and the brain surface was approximately $1 \mathrm{~mm}$ or less. In this feasibility study, we preferred closer distance to maintain the level of resolution and effectively collect returned signals for OCT reconstruction.

\section{Results and discussion}

Figure 2 is presented to demonstrate the feasibility of our method based on time-domain reflectometry CP-OCT. The different tissue regions are directly compared in Fig. 2a: the layer appears thicker in the tumor tissue (left-hand side of the image), and the normal tissue (right-hand side of the image) has a thin bright surface. As can be observed, tumor tissue scatters light more strongly at greater depths below the surface, compared with the normal tissue. In contrast, the latter displays abrupt reflection at the surface because of its smoothness. A discontinuity between the surface topology of the tumor and normal tissues can also be observed, and this facilitates the differentiation of the two significant regions [7]. In addition, a full-image view of the tumor in the mouse brain is displayed in Fig. $2 \mathbf{b}$ for comparison, providing a clear illustration of the strongly scattering internal tissue within the tumor region. Here, due to the size 

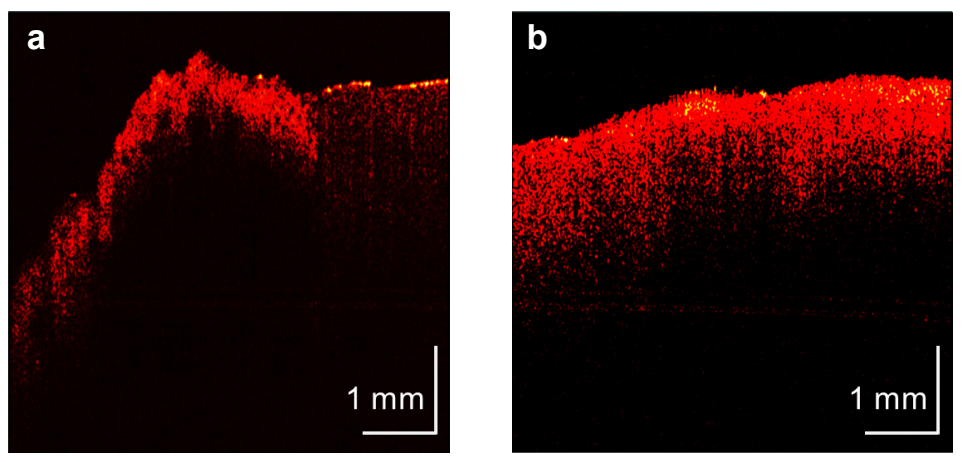

Fig. 2. Results of cross-sectional views of mouse brain tissues: regional view of normal tissue and adjacent tumor (a), and regional view of tumor tissue (b).

of the imaging area, the length of the sweeping optical fiber can be kept short in order to avoid and/or minimize the fiber fluctuation while scanning directly without the need of providing extra rigidness of the optical fiber itself. The lateral resolution is proportional to the distance based on the diffraction beam of the employed fiber probe. In out experiment, the lateral resolution is approximately $45 \mu \mathrm{m}$.

Furthermore, the resection areas were imaged after the removal of a small area of tumor in two different sample areas, as shown in Fig. 3. Owing to the high-resolution of OCT imaging, it was possible to detect the resected areas as sharp grooves formed by the surgical blades. Using our compact and simple system, real-time and in situ images of the resection in challenging and delicate brain regions were provided. Thus, in addition to the preoperative images, these intraoperative images can precisely guide the surgeon, allowing greater capacity to monitor the surgical procedure during delicate operations and hence minimizing the risk of surgical complications. Further practical issues could be alleviated by installing depth locking and/or surface tracking systems to avoid damage to the tissue $[\underline{8}]$.
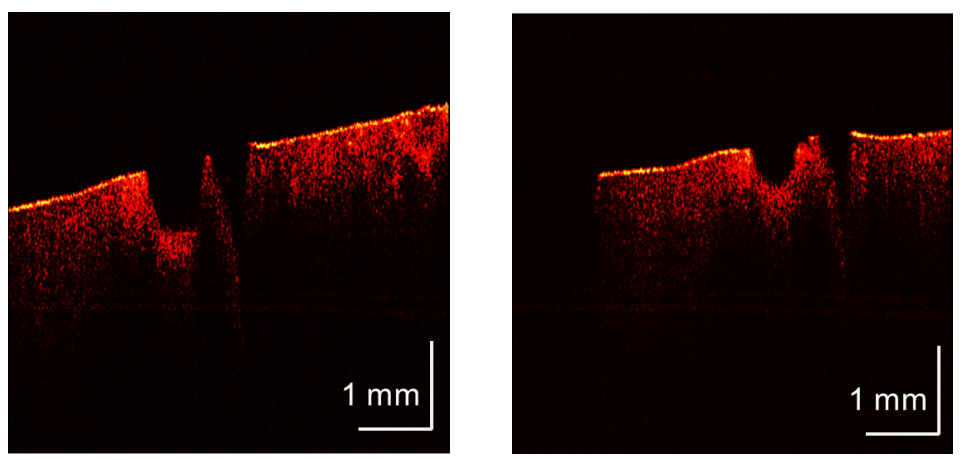

Fig. 3. Results after tumor resection: two different areas of brain imaged after removal of a tumor (the grooved region indicates the former location of the tumor). 


\section{Conclusions}

In summary, rapid imaging of a mouse brain tumor was demonstrated by $1.3-\mu \mathrm{m}$ near -infrared time-domain reflectometric CP-OCT using a galvanometer attached to a bare -fiber probe. Common-path optical interferometry was used to scan a wide area in real -time, with the same probe providing the reference signal, resulting in a simpler system that allows the use of a disposable probe and freely adjustable probe length. The results reveal that visual differentiation of the features of the brain tumor and normal tissue is possible. Furthermore, the acquired images have resolution sufficient to clearly show in situ resection areas in brain surgery. Though the possible limitations under the proposed method, this approach should contribute to the advancement of high-performance minimally invasive intraoperative imaging for microsurgical image-guided interventions.

Acknowledgements - This work was supported in part by the NRF grant funded by the Korean government (NRF-2017R1A2B2003808). This work was also supported in part by LG Yonam Foundation (of Korea).

\section{References}

[1] Сha J., Broch A., Mudge S. Kim K., Namgoong J., Оh E., Kim P., Real-time, label-free, intraoperative visualization of peripheral nerves and micro-vasculatures using multimodal optical imaging techniques, Biomedical Optics Express 9(3), 2018, pp. 1097-1110, DOI: 10.1364/BOE.9.001097.

[2] Garzon-Muvdi T., Kut C., Li X., Chaichana K.L., Intraoperative imaging techniques for glioma surgery, Future Oncology 13(19), 2017, pp. 1731-1745, DOI: 10.2217/fon-2017-0092.

[3] Keating J., Tchou J., Okusanya O., Fisher C., Batiste R., Jiang J., Kennedy G., Nie S., Singhal S., Identification of breast cancer margins using intraoperative near-infrared imaging, Journal of Surgical Oncology 113(5), 2016, pp. 508-514, DOI: 10.1002/jso.24167.

[4] Lee S., Lee C., Verkade R., Cheon G.W., Kang J.U., Common-path all-fiber optical coherence tomography probe based on high-index elliptical epoxy-lensed fiber, Optical Engineering 58(2), 2019, article 026116, DOI: 10.1117/1.OE.58.2.026116.

[5] Katta N., Mcelroy A.B., Estrada A.D., Milner T.E., Optical coherence tomography image-guided smart laser knife for surgery, Lasers in Surgery and Medicine 50(3), 2018, pp. 202-212, DOI: 10.1002/ $\underline{1 \mathrm{sm} .22705 .}$.

[6] Li X., HAN J.-H., LiU X., KANG J.U., Signal-to-noise ratio analysis of all-fiber common-path optical coherence tomography, Applied Optics 47(27), 2008, pp. 4833-4840, DOI: 10.1364/AO.47.004833.

[7] Böhringer H.J., Lankenau E., Stellmacher F., Reusche E., Hüttmann G., Giese A., Imaging of human brain tumor tissue by near-infrared laser coherence tomography, Acta Neurochirurgica 151(5), 2009, pp. 507-517, DOI: 10.1007/s00701-009-0248-y.

[8] Cheon G.W., Huang Y., Cha J., Gehlbach P.L., Kang J.U., Accurate real-time depth control for CP-SSOCT distal sensor based handheld microsurgery tools, Biomedical Optics Express 6(5), 2015, pp. 1942-1953, DOI: $10.1364 /$ BOE.6.001942. 